\title{
Periodic finite-genus solutions of the KdV equation are orbitally stable
}

\author{
Michael Nivala and Bernard Deconinck \\ Department of Applied Mathematics, University of Washington, \\ Campus Box 352420, Seattle, WA, 98195, USA
}

December 1, 2009

\begin{abstract}
The stability of periodic solutions of partial differential equations has been an area of increasing interest in the last decade. The KdV equation is known to have large families of periodic solutions that are parameterized by hyperelliptic Riemann surfaces. They are generalizations of the famous multi-soliton solutions. We show that all such periodic solutions are orbitally stable with respect to subharmonic perturbations: perturbations that are periodic with period equal to an integer multiple of the period of the underlying solution.
\end{abstract}

\section{Keywords: Stability, Korteweg-de Vries equation, periodic solutions PACS codes: 02.30.Ik, 02.30.Jr, 47.10.Fg}

\section{Introduction}

The Korteweg-deVries (KdV) equation

$$
u_{t}+u u_{x}+u_{x x x}=0,
$$

describes long, one-dimensional waves in weakly dispersive media and arises in a variety of physical settings ranging from water waves to plasma physics [33]. It is characterized by its trademark soliton solutions and their quasi-periodic analogues. The most explicit of these are the one-soliton solution

$$
u=u_{0}+12 \kappa^{2} \operatorname{sech}^{2}\left(\kappa\left(x-x_{0}-\left(4 \kappa^{2}+u_{0}\right) t\right)\right),
$$

and its periodic counterpart, the cnoidal wave solution

$$
u=u_{0}+12 k^{2} \kappa^{2} \operatorname{cn}^{2}\left(\kappa\left(x-x_{0}-\left(8 \kappa^{2} k^{2}-4 \kappa^{2}+u_{0}\right) t\right), k\right),
$$

both of which were known to Korteweg and deVries [38]. Here $u_{0}, \kappa$, and $x_{0}$ are constants, and $\operatorname{cn}(\cdot, k)$ denotes the Jacobi elliptic cosine function $[15,41]$ with elliptic modulus $k \in[0,1)$.

The stability problem for the above solutions has a rich history (a more detailed discussion is found in [12]), beginning with the works of Benjamin and Bona [8, 11], where the nonlinear orbital stability of the one-soliton solution (1.2) with respect to $L^{2}$ perturbations was established. Later, Maddocks and Sachs generalized this result for general multi-soliton solutions [43]. More recently, 
the methods used by Benjamin and Bona were extended to the periodic problem, and the nonlinear orbital stability of cnoidal waves (1.3) with respect to periodic perturbations of the same period was verified [4]. Going beyond periodic perturbations of the same period, Bottman and Deconinck proved the spectral stability of cnoidal waves with respect to bounded perturbations [12], and in a follow-up manuscript with Kapitula, the orbital stability of cnoidal waves with respect to subharmonic perturbations (periodic perturbations with period equal to any integer multiple of the period of the cnoidal wave) was established [16]. These papers rely, at least partially, on the integrability of the $\mathrm{KdV}$ equation. Alternatively, the $\mathrm{KdV}$ equation has been approached as a special case of the generalized KdV equation, which allows for more general nonlinearities. Different authors have developed different techniques for the investigation of the stability of stationary periodic solutions of the generalized KdV equation (see, for instance, $[13,14,35]$ ). These techniques are more general as they apply to larger classes of equations and do not rely on integrability. On the other hand, the results they provide are less detailed than what is obtained using integrability.

In this paper we are concerned with the stability of the periodic analogs of the multi-soliton solutions, the periodic finite-genus solutions. These are a large family of periodic solutions with $n$ phases of the form $[7,21,34,39]$

$$
u(x, t)=u_{0}+2 \partial_{x}^{2} \ln \Theta\left(\phi_{1}, \ldots, \phi_{n} \mid B\right),
$$

where each phase $\phi_{j}$ is linear in $x$ and $t$,

$$
\phi_{j}=k_{j} x+\omega_{j} t+\phi_{0 j},
$$

for constants $u_{0}$ and $k_{j}, w_{j}, \phi_{0 j}, j=1, \ldots, n .{ }^{1}{ }^{2}$ Here $\Theta$ denotes the Riemann theta function [24], parametrized by the Riemann matrix $B$ which is determined by a genus $n$ connected compact hyperelliptic Riemann surface generated by the initial condition $u(x, 0)$. The details of the theta function formulation are of little importance to us here, as they are not used in what follows. It should be noted that in the case $n=1$ the solution (1.4) is equivalent to the cnoidal wave solution (1.3). The periodic finite-genus solutions possess the following properties:

- They completely solve the initial-value problem for the KdV equation with periodic boundary conditions in the following sense: (i) They solve the initial-value problem for initial data that are periodic in $x$, and are of the form $(1.4)[9,22,42,48]$. (ii) They are dense in the set of smooth periodic functions [46]. Therefore, the stability of periodic finite-genus initial conditions in a suitable function space suggests the stability of general periodic initial conditions in that function space.

- Their stability was studied first by McKean [45]. He established that the tori on which the periodic finite-genus solutions lie are stable with respect to periodic perturbations. As noted in [43], McKean only briefly discusses the implications of his results concerning stability in a normed space, such as $L^{2}$.

The object of this paper is to establish the (nonlinear) orbital stability of the periodic finitegenus solutions with respect to subharmonic perturbations. Extension beyond periodic perturbations of the same period to subharmonic perturbations is important in that: (i) they are a

\footnotetext{
${ }^{1}$ A genus $n$ solution is periodic in $x$ with period $2 L$ if there exist $n$ integers $N_{1}, \ldots, N_{n}$ such that $2 L k_{i}=2 \pi N_{i}$ for $i=1, \ldots, n$, otherwise, it is quasi-periodic.

${ }^{2}$ We restrict ourselves to real-valued finite-genus solutions.
} 
significantly larger class of perturbations than the periodic ones of the same period, while retaining our ability to discuss completeness and separability of a suitable function space. For example, this would be more complicated for the case for quasi-periodic or almost periodic perturbations [10]. (ii) There are nontrivial examples of solutions which are stable with respect to periodic perturbations of the same period, but unstable with respect to subharmonic perturbations, such as the trivialphase cnoidal wave solutions of the focusing nonlinear Schrödinger equation [28]. (iii) They have a greater physical relevance than periodic perturbations of the same period, since in applications one usually considers domains which are larger than the period of the solution, e.g., ocean wave dynamics.

The basis of our procedure is the Lyapunov method, which was first extended to infinitedimensional systems (partial differential equations) by V.I. Arnold [5, 6] in his study of incompressible ideal fluid flows. Since its introduction, the Lyapunov method has formed the crux of subsequent nonlinear stability techniques (see $[29,30,52]$ for instance). We build on the results recently obtained for the cnoidal wave (genus one) solutions in [16], and present a systematic generalization. As in [16], the method relies heavily on the integrability of the KdV equation. The outline of the method is as follows:

- Each genus $n$ solution (1.4) is a stationary solution of the $n$-th KdV equation (to be defined later) with evolution variable $t_{n}$ [39]. In turn, every bounded periodic stationary solution of the $n$-th $\mathrm{KdV}$ equation is a genus $n$ solution of the form (1.4) [9, 22, 42, 48]. Our method does not require nor make use of the explicit form of the solution (1.4). We only require that it is stationary (with respect to the higher-order time variable $t_{n}$ ) and periodic in $x$.

- Since a genus $n>1$ solution is not stationary with respect to the KdV flow itself, we cannot define spectral stability in the conventional sense. Instead we prove spectral stability with respect to bounded perturbations (not necessarily periodic) for the higher-order time variable $t_{n}$. We do this by generalizing the squared eigenfunction method used in [12] for the cnoidal wave solutions.

- We use the ideas in $[16,43]$ to construct a candidate Lyapunov function. We show that it is indeed a Lyapunov function using the squared eigenfunction connection and the spectral stability result. This establishes orbital stability, using the results from [26, 27].

We also look at some explicit examples, including comparison with numerical results using Hill's method $[17,18]$. It is interesting to point out that the multi-soliton solutions can be obtained from (1.4) by taking certain limits [22]. In that case our method (informally) recovers the stability results in [43].

Remark. Though we present the method for finite-genus solutions of the KdV equation, the main ideas carry over to other integrable systems, i.e., the general steps and principles still apply, but the details will be different.

\section{Problem formulation}

\subsection{Hamiltonian structure}

We are concerned with the stability of $2 L$-periodic genus $n$ solutions of equation (1.1) with respect to subharmonic perturbations of period $2 N L$ for any fixed positive integer $N$. Therefore, we naturally 
consider solutions $u$ in the space of square-integrable functions of period $2 N L, L_{p e r}^{2}[-N L, N L]$. In order to properly define the Hamiltonian structure of the KdV equation and the corresponding KdV hierarchy (see Section 2.2), we further require $u$ and its derivatives of order up to $2 n+1$ to be square-integrable as well. Therefore, we consider solutions of (1.1) defined on the function space

$$
\mathbb{V}=H_{p e r}^{2 n+1}[-N L, N L],
$$

equipped with the natural inner product

$$
\langle v, w\rangle=\int_{-N L}^{N L} \bar{v} w d x
$$

where $\bar{v}$ denotes the complex conjugate of $v$.

We write equation (1.1) in Hamiltonian form [25, 53]

$$
u_{t}=J H^{\prime}(u)
$$

on $\mathbb{V}$. Here $J$ is the skew-symmetric operator

$$
J=\partial_{x}
$$

the Hamiltonian $H$ is the functional

$$
H(u)=\int_{-N L}^{N L}\left(\frac{1}{2} u_{x}^{2}-\frac{1}{6} u^{3}\right) d x
$$

and the notation $E^{\prime}$ denotes the variational derivative of $E=\int_{-N L}^{N L} \mathcal{E}\left(u, u_{x}, \ldots\right) d x$,

$$
E^{\prime}(u)=\sum_{j=0}^{\infty}(-1)^{j} \partial_{x}^{j} \frac{\partial \mathcal{E}}{\partial u_{j x}},
$$

where the sum in (2.3) terminates at the order of the highest derivative involved. For instance, in the computation of $H^{\prime}$ the sum terminates after accounting for first-derivative terms.

We allow for perturbations in a function space $\mathbb{V}_{0} \subset \mathbb{V}$. In order to apply the stability result of [27], we follow [16] and restrict ourselves to the space of perturbations on which $J$ has a welldefined and bounded inverse. This amounts to fixing the spatial average of $u$ on $H_{p e r}^{2 n+1}[-N L, N L]$, which poses no problem since it is a Casimir of the Poisson operator $J$, hence, it is conserved under the $\mathrm{KdV}$ flow. Therefore, we consider perturbations in $\operatorname{ker}(J)^{\perp}$, i.e., zero-average subharmonic perturbations

$$
\mathbb{V}_{0}=\left\{v \in H_{p e r}^{2 n+1}([-N L, N L]): \frac{1}{2 N L} \int_{-N L}^{N L} v d x=0\right\}
$$

Remark. Physically, requiring perturbations to have zero-average makes sense. Simply stated, this means we are not allowing perturbations to add or subtract mass from the system. 


\subsection{The nonlinear KdV hierarchy}

By virtue of its integrability, the $\mathrm{KdV}$ equation possesses an infinite number of conserved quantities

[40] $H_{0}, H_{1}, H_{2}, \ldots$, and just as the functional $H_{1}=H$ defines the KdV equation, each $H_{j}$ defines a Hamiltonian system with time variable $\tau_{j}$ through

$$
u_{\tau_{j}}=J H_{j}^{\prime}(u)
$$

The first few conserved quantities are

$$
\begin{aligned}
H_{0} & =\int_{-N L}^{N L} \frac{1}{2} u^{2} d x \\
H_{1} & =H=\int_{-N L}^{N L}\left(\frac{1}{2} u_{x}^{2}-\frac{1}{6} u^{3}\right) d x \\
H_{2} & =\int_{-N L}^{N L}\left(\frac{1}{2} u_{x x}^{2}-\frac{5}{6} u u_{x}^{2}+\frac{5}{72} u^{4}\right) d x
\end{aligned}
$$

with corresponding flows

$$
\begin{aligned}
& u_{\tau_{0}}=u_{x} \quad\left(\text { identifies } \tau_{0} \text { with } x\right), \\
& u_{\tau_{1}}=-u u_{x}-u_{x x x} \quad\left(\text { identifies } \tau_{1} \text { with } t\right), \\
& u_{\tau_{2}}=\frac{5}{6} u^{2} u_{x}+\frac{10}{3} u_{x} u_{x x}+\frac{5}{3} u u_{x x x}+u_{x x x x x} .
\end{aligned}
$$

Each of the higher-order flows can be explicitly calculated from the recursion formula (see [49], for instance)

$$
u_{\tau_{j+1}}=\mathcal{R} u_{\tau_{j}}, \quad u_{\tau_{0}}=u_{x}
$$

where $\mathcal{R}$ is the operator

$$
\mathcal{R}=-\partial_{x}^{2}-\frac{2}{3} u-\frac{1}{3} u_{x} \partial_{x}^{-1}
$$

Since each $u_{\tau_{j}}$ is a total derivative, the non-local term $\partial_{x}^{-1}$ in $(2.6)$ is well defined [42, 49]. This defines an infinite hierarchy of equations, the KdV hierarchy. It has the following properties:

- All the functionals $H_{j}, j=0,1, \ldots$, are conserved for each member of the KdV hierarchy (2.5) [42].

- The flows of the KdV hierarchy (2.5) mutually commute, and we can think of $u$ as solving all of these equations simultaneously, i.e., $u=u\left(\tau_{0}, \tau_{1}, \ldots\right)[20,42]$.

- A finite-genus solution (1.4) of the $\mathrm{KdV}$ equation is a simultaneous solution of all the flows of the KdV hierarchy [50], where now the phases depend on all the time variables

$$
\phi_{j}=\sum_{i=0}^{\infty} k_{j, i} \tau_{i} .
$$


For a genus $n$ solution, dependence on all but a finite number of the higher-order time variables is trivial. This is discussed further in Section 2.3.

Since all the flows of the KdV hierarchy commute, we may take any linear combination of the above Hamiltonians to define a new Hamiltonian system. For our purposes, we define the $n$-th $K d V$ equation with time variable $t_{n}$ as

$$
u_{t_{n}}=J \hat{H}_{n}^{\prime}(u)
$$

where each $\hat{H}_{n}$ is defined as

$$
\hat{H}_{n}:=H_{n}+\sum_{j=0}^{n-1} c_{n, j} H_{j}, \quad \hat{H}_{0}:=H_{0},
$$

for constants $c_{n, j}, j=0, \ldots, n-1$.

\section{Remarks.}

- No constraints have been imposed on the parameters $c_{n, j}$. We use this freedom to our advantage below.

- There are two hierarchies of equations considered here. The hierarchy associated with the $t_{j}$ time variables contains the one associated with the $\tau_{j}$ time variables as a special case.

- The time variable $t_{0}$ remains identified with $x$.

- We did not include the conserved quantity $H_{-1}=\int_{-N L}^{N L} u d x$ in any of the above. Its variational derivative is constant and thus $H_{-1}$ spans the kernel of $J$. This results in trivial dynamics. $H_{-1}$ is known as a Casimir. As discussed in [16] and seen in (2.4), the existence of such a functional restricts the space of allowed perturbations.

\subsection{Stationary solutions of the KdV hierarchy}

It was first shown in $[22,42,48]$ that the $\mathrm{KdV}$ equation possesses a large class of periodic and quasi-periodic solutions by examining stationary solutions of the $n$-th $\mathrm{KdV}$ equation (2.7). These are solutions for which

$$
u_{t_{n}}=0 \text {, }
$$

for some integer $n$ and constants $c_{n, 0}, \ldots, c_{n, n-1}$ in (2.7-2.8). Thus, a stationary solution of the $n$-th KdV equation satisfies the ordinary differential equation

$$
J \hat{H}_{n}^{\prime}(u)=0
$$

with independent variable $x$.

We are interested in the stability of the finite-genus solutions (1.4). This is equivalent to the study of the stationary solutions of the $\mathrm{KdV}$ hierarchy by the following theorem $[42,48,50]$ :

Theorem. Each genus $n$ solution (1.4) is a stationary solution of the $n$-th KdV equation (2.7) for some choice of the constants $c_{n, 0}, \ldots, c_{n, n-1}$. In turn, every bounded stationary solution of the 
$n$-th KdV equation is a genus $n$ solution of the form (1.4), or a limit of such a solution (e.g., the soliton solutions).

Here bounded means that $\sup _{x \in \mathbb{R}}|u(x)|$ is finite, i.e., $u(x) \in C_{b}^{0}(\mathbb{R})$.

Throughout the rest of the paper we use the terms stationary solution of the $n$-th KdV equation and genus $n$ solution interchangeably. Also, when referring to a genus $n$ solution $u$ we assume it is non-degenerate, i.e., $u$ is a stationary solution of the $n$-th $\mathrm{KdV}$ equation and is not stationary with respect to any of the lower-order flows.

The stationary solutions of the KdV hierarchy have the following properties.

- Since all the flows commute, the set of stationary solutions is invariant under any of the KdV equations, i.e., a stationary solution of the $n$-th $\mathrm{KdV}$ equation remains a stationary solution of the $n$-th KdV equation after evolving under any of the other flows [42].

- Any stationary solution of the $n$-th $\mathrm{KdV}$ equation is also stationary with respect to all of the higher order time variables $t_{m}, m>n$. In such cases, the constants $c_{m, i}, i \geq n$ are free parameters. We make use of this fact when constructing a Lyapunov function later.

Example. The first flow with time variable $t_{1}(2.7)$ is given by

$$
u_{t_{1}}=-u u_{x}-u_{x x x}+c_{1,0} u_{x}
$$

Looking for stationary solutions, i.e., setting $u_{t_{1}}=0$ results in the ordinary differential equation

$$
-u u_{x}-u_{x x x}+c_{1,0} u_{x}=0 .
$$

All periodic solutions of this equation can be written as [38]

$$
u=c_{1,0}-8 \kappa^{2} k^{2}+4 \kappa^{2}+12 k^{2} \kappa^{2} \operatorname{cn}^{2}\left(\kappa\left(x-x_{0}\right), k\right),
$$

where $x_{0}$ and $\kappa$ are arbitrary constants due to translation and scaling symmetries. The period $2 L$ is given by

$$
2 L=\frac{2 \mathrm{~K}(k)}{\kappa}=\frac{2}{\kappa} \int_{0}^{\pi / 2} \frac{1}{\sqrt{1-k^{2} \sin ^{2} s}} d s,
$$

where $\mathrm{K}(k)$ is the complete elliptic integral of the first kind $[15,41]$. Using the Galilean invariance of the KdV equation, we recover the well-known cnoidal wave solution (1.3).

To see the other side of the previous theorem, suppose we are given a genus one initial condition

$$
u^{*}(x)=12 k^{2} \mathrm{cn}^{2}(x, k) .
$$

Imposing that $u^{*}$ is stationary with respect to $t_{1}$ fixes $c_{1,0}$ as

$$
c_{1,0}=8 k^{2}-4
$$

Furthermore, we can fix all constants $c_{m, 0}, m \geq 1$, such that $u^{*}$ is stationary with respect to all the higher-order time variables $t_{m}$. For example, imposing that $u^{*}$ is a stationary solution of the second KdV equation, 


$$
0=u_{t_{2}}=\frac{5}{6} u^{2} u_{x}+\frac{10}{3} u_{x} u_{x x}+\frac{5}{3} u u_{x x x}+u_{x x x x x}+c_{2,1}\left(-u u_{x}-u_{x x x}\right)+c_{2,0} u_{x},
$$

fixes $c_{2,0}$ as

$$
c_{2,0}=c_{2,1}\left(8 k^{2}-4\right)-56 k^{4}+56 k^{2}-16 .
$$

Thus, $c_{2,1}$ is a free parameter, and $u^{*}$ is a stationary solution of the second KdV equation for any value of $c_{2,1}$ with $c_{2,0}$ defined as above.

\section{Remarks.}

- In general, finite-genus solutions are quasi-periodic in time as opposed to periodic, even if they are periodic in $x[42]$.

- The soliton solutions are obtained as a special case of the finite-genus solutions [22]. For example, the one-soliton (1.2) is obtained form the cnoidal wave (1.3) by letting $k \rightarrow 1$.

\subsection{Stability}

We assume our solution is a stationary solution of the $n$-th flow

$$
u\left(x, t_{1}, \ldots, t_{n-1}, t_{n}\right)=u^{*}\left(x, t_{1}, \ldots, t_{n-1}\right)
$$

and consider various aspects of stability. Since we are considering $u$ as a solution of the first $n$ equations in the KdV hierarchy, we have suppressed dependence on the higher-order time variables $t_{i}, i>n$, in (2.13). Linearizing the $n$-th $\mathrm{KdV}$ equation (2.7) about $u^{*}$,

$$
u\left(x, t_{1}, \ldots, t_{n}\right)=u^{*}+\epsilon w\left(x, t_{1}, \ldots, t_{n}\right)+\mathcal{O}\left(\epsilon^{2}\right),
$$

results in the linear system

$$
w_{t_{n}}=J \mathcal{L}_{n} w .
$$

Here the symmetric differential operator $\mathcal{L}_{n}:=\hat{H}_{n}^{\prime \prime}\left(u^{*}\right)$ is the Hessian of $\hat{H}_{n}$,

$$
\hat{H}_{n}^{\prime \prime}(u)=\sum_{j=0}^{\infty} \frac{\partial \hat{H}_{n}^{\prime}(u)}{\partial u_{j x}} \partial_{x}^{j},
$$

evaluated at the stationary solution (as before, the above sum terminates at the order of the highest derivative involved). The operator $\mathcal{L}_{n}$ does not depend on $t_{n}$, thus we separate variables

$$
w\left(x, t_{n}\right)=e^{\lambda_{n} t_{n}} W(x) .
$$

Since the operator $\mathcal{L}_{n}$ is expressed solely in terms of $x$, we have suppressed dependence on the lower-order time variables $t_{1}, \ldots, t_{n-1}$. This results in the spectral problem

$$
J \mathcal{L}_{n} W(x)=\lambda_{n} W(x)
$$


Definition. We say the solution $u^{*}$ is $t_{n}$-spectrally stable with respect to perturbations in a function space $\mathbb{U}$ if $\operatorname{Re}\left(\lambda_{n}\right) \leq 0$ for all $W \in \mathbb{U}$.

For Hamiltonian systems $t_{n}$-spectral stability is equivalent to $\lambda_{n} \in i \mathbb{R}$. We define the stability spectrum $\sigma\left(J \mathcal{L}_{n}\right)$ as the spectrum of the operator $J \mathcal{L}_{n}$.

Example. Linearizing (2.9) about $u=u^{*}$ from (2.11) results in the spectral problem

$$
J \mathcal{L}_{1} W(x)=\lambda_{1} W(x)
$$

where $\mathcal{L}_{1}$ is given by

$$
\hat{H}_{1}^{\prime \prime}\left(u^{*}\right)=-\partial_{x}^{2}+c_{1,0}-u^{*}
$$

with $c_{1,0}$ defined in (2.12).

Now, consider the problem of nonlinear stability. The $n$-th KdV equation is invariant under translation with respect to any of the lower-order time variables. This is represented by the Lie group

$$
G=\mathbb{R}^{n}
$$

which acts on $u\left(x, \ldots, t_{n}\right)$ according to

$$
T(g) u\left(x, \ldots, t_{i}, \ldots, t_{n-1}, t_{n}\right)=u\left(x+t_{00}, \ldots, t_{i}+t_{0 i}, \ldots, t_{n-1}+t_{0(n-1)}, t_{n}\right)
$$

with $g=\left(t_{00}, \ldots, t_{0(n-1)}\right) \in G$. Stability is considered modulo this symmetry.

Definition. A stationary solution $u^{*}$ of the $n$-th $\mathrm{KdV}$ equation is orbitally stable under the $t_{j}$ dynamics with respect to perturbations in $\mathbb{V}_{0}$ if for a given $\epsilon>0$ there exists a $\delta>0$ such that

$$
\begin{aligned}
& \left\|u\left(x, \ldots, t_{j-1}, 0, t_{j+1}, \ldots\right)-u^{*}\left(x, \ldots, t_{n-1}\right)\right\|<\delta \\
\Rightarrow \quad & \inf _{g \in G}\left\|u\left(x, \ldots, t_{j}, \ldots\right)-T(g) u^{*}\left(x, \ldots, t_{n-1}\right)\right\|<\epsilon,
\end{aligned}
$$

where $\|\cdot\|$ denotes the norm obtained through $\langle\cdot, \cdot\rangle$ on $\mathbb{V}_{0}$.

One can think of the above definition as allowing for the optimal variation of the $n$ phase constants in (1.4) before measuring the distance between functions. Thus, our definition of orbital stability of finite-genus solutions with periodic boundary conditions is equivalent to the analogous version in [43] for $n$-solitons with vanishing boundary conditions.

To prove orbital stability, we search for a Lyapunov function. For Hamiltonian systems, this is a constant of the motion, $E(u)$, for which $u^{*}$ is an unconstrained minimum:

$$
\partial_{t} E(u)=0, \quad E^{\prime}\left(u^{*}\right)=0, \quad\left\langle v, E^{\prime \prime}\left(u^{*}\right) v\right\rangle>0, \quad \forall v \in \mathbb{V}_{0}, v \neq 0 .
$$

The existence of such a function implies formal stability [30]. Due to the work of Grillakis, Shatah, and Strauss [26], under extra conditions (see the orbital stability theorem below) this allows one to conclude orbital stability. Since all the KdV flows mutually commute, orbital stability with respect 
to any of the time variables $t_{i}$ implies stability with respect to all of the flows of the KdV hierarchy, most importantly the KdV flow [16, 36, 43], as the Lyapunov function serves the same role for each equation in the hierarchy.

Just as the norm of the difference of two solutions is considered modulo symmetries, in effect the same is done when considering a Lyapunov function. Using the Lyapunov function construction techniques from $[16,43]$, we have the following stability theorem due to [26]:

Theorem. Let $u^{*}$ be a $t_{n}$-spectrally stable stationary solution of equation (2.7) such that the eigenfunctions $W$ of the linear stability problem (2.16) form a basis for the space of allowed perturbations $\mathbb{V}_{0}$, on which $J$ has bounded inverse. Furthermore, suppose there exists an integer $m>n$ and constants $c_{m, 0}, \ldots, c_{m, m-1}$ such that the Hamiltonian for the $m$-th KdV equation satisfies:

1. The kernel of $\hat{H}_{m}^{\prime \prime}\left(u^{*}\right)$ on $\mathbb{V}_{0}$ is spanned by the infinitesimal generators of the symmetry group $G$ acting on $u^{*}$.

2. For all eigenfunctions not in the kernel of $\hat{H}_{m}^{\prime \prime}\left(u^{*}\right)$

$$
K_{m}(W):=\left\langle W, \hat{H}_{m}^{\prime \prime}\left(u^{*}\right) W\right\rangle>0 .
$$

Then $u^{*}$ is orbitally stable under the $t_{i}$ dynamics, $i=1,2, \ldots$, with respect to perturbations in $\mathbb{V}_{0}$.

\section{Remarks.}

- The last condition $\left\langle W, \hat{H}_{m}^{\prime \prime}\left(u^{*}\right) W\right\rangle>0$ can be replaced with $\left\langle W, \hat{H}_{m}^{\prime \prime}\left(u^{*}\right) W\right\rangle<0$. In this case $-\hat{H}_{m}(u)$ serves as a Lyapunov function. Thus, we only need that $\left\langle W, \hat{H}_{m}^{\prime \prime}\left(u^{*}\right) W\right\rangle$ is of definite sign.

- Often, the sign of $K_{n}$ is called the Krein signature, see [37].

- For spectral stability we only require perturbations to be spatially bounded, thus $\mathbb{U}=C_{b}^{0}(\mathbb{R})$.

\section{Spectral Stability}

We prove that a periodic stationary solution of the $n$-th $\mathrm{KdV}$ equation is $t_{n}$-spectrally stable with respect to bounded perturbations. In order to accomplish this, we use the relationship between the solution of the Lax pair equations and the solution of the linear stability problem, the squared eigenfunction connection $[2,12,42]$.

\subsection{The Lax pair and the linear hierarchy}

The KdV equation is equivalent to the compatibility of two linear ordinary differential systems

$$
\begin{aligned}
& \psi_{x}=T_{0} \psi=\left(\begin{array}{cc}
0 & 1 \\
\zeta-u / 6 & 0
\end{array}\right) \psi \\
& \psi_{t}=T_{1} \psi=\left(\begin{array}{cc}
u_{x} / 6 & -4 \zeta-u / 3 \\
-4 \zeta^{2}+\left(u^{2}+6 \zeta u+3 u_{x x}\right) / 18 & -u_{x} / 6
\end{array}\right) \psi
\end{aligned}
$$


In other words, the compatibility condition $\psi_{x t}=\psi_{t x}$ implies that $u$ satisfies the KdV equation. Equation (3.1) is equivalent to

$$
\partial_{x x} \psi_{1}+\frac{1}{6} u \psi_{1}=\zeta \psi_{1} .
$$

Therefore, $\zeta$ is the spectral parameter for the stationary Schrödinger equation. This implies $\zeta \in \mathbb{R}$ for any bounded solution of (3.3).

Since every member of the nonlinear hierarchy (2.5) is integrable, each possesses a Lax pair with first member (3.1), the collection of which is known as the linear KdV hierarchy. For example, the second Lax equation associated with the time variable $\tau_{2}$ is

$$
\psi_{\tau_{2}}=\left(\begin{array}{cc}
-\frac{1}{6}\left(u u_{x}+u_{x x x}\right)-\frac{2}{3} \zeta u_{x} & 16 \zeta^{2}+\frac{4}{3} \zeta u+\frac{1}{6} u^{2}+\frac{1}{3} u_{x x} \\
16 \zeta^{3}-\frac{4}{3} \zeta^{2} u-\zeta\left(\frac{1}{18} u^{2}+\frac{1}{3} u_{x x}\right) & \\
-\frac{1}{36} u^{3}-\frac{1}{6} u_{x}^{2}-\frac{2}{9} u u_{x x}-\frac{1}{6} u_{x x x x} & \frac{1}{6}\left(u u_{x}+u_{x x x}\right)+\frac{2}{3} \zeta u_{x}
\end{array}\right) \psi
$$

All the higher order Lax operators are calculated in standard fashion, see $[1,2]$ for instance. We include some of the details here because our later calculations rely on them. Assume the time component of the Lax pair for the $n$-th flow with time variable $\tau_{n}$ takes the form

$$
\psi_{\tau_{n}}=T_{n} \psi=\left(\begin{array}{cc}
A_{n} & B_{n} \\
C_{n} & -A_{n}
\end{array}\right) \psi
$$

Expressing the compatibility $\psi_{\tau_{n} x}=\psi_{x \tau_{n}}$ gives

$$
\begin{aligned}
A_{n} & =-\frac{1}{2} \partial_{x} B_{n} \\
C_{n} & =\partial_{x} A_{n}+\left(\zeta-\frac{1}{6} u\right) B_{n} \\
u_{\tau_{n}} & =(12 \zeta-2 u) A_{n}-6 \partial_{x} C_{n} .
\end{aligned}
$$

Solving the first two equations for $A_{n}$ and $C_{n}$, we can express the last equation solely in terms of $B_{n}$ :

$$
u_{\tau_{n}}=-12 \zeta \partial_{x} B_{n}+2 u \partial_{x} B_{n}+u_{x} B_{n}+3 \partial_{x}^{3} B_{n} .
$$

The $n$-th member of the hierarchy is found by assuming an expansion of the form

$$
B_{n}=\sum_{j=0}^{n} b_{j}(x) \zeta^{j} .
$$

Substituting this assumption into (3.4) gives $b_{n}(x)=b_{n}$, a constant, and the recursive relationship

$$
b_{j-1}(x)=\int\left(\frac{1}{4} \partial_{x}^{3} b_{j}(x)+\frac{1}{6} u \partial_{x} b_{j}(x)+\frac{1}{12} u_{x} b_{j}(x)\right) d x,
$$

which will be of use later. It can be shown that the integrand in (3.6) is always a total derivative, thus each $T_{n}$ depends on $u$ and its derivatives in a purely local fashion [42, 49]. 
We construct the Lax pair for the $n$-th KdV equation (2.7) by taking the same linear combination of the lower-order flows as we did for the Hamiltonians (2.8), and define the $n$-th linear KdV equation as

$$
\begin{gathered}
\psi_{t_{n}}=\hat{T}_{n} \psi=\left(\begin{array}{cc}
\hat{A}_{n} & \hat{B}_{n} \\
\hat{C}_{n} & -\hat{A}_{n}
\end{array}\right) \psi, \\
\hat{T}_{n}:=T_{n}+\sum_{j=0}^{n-1} c_{n, j} T_{j}, \quad \hat{T}_{0}:=T_{0} .
\end{gathered}
$$

Note that the extra constant $b_{n}$ from the leading order term in the expansion for $B_{n}(3.5)$ is chosen so that the compatibility condition gives (2.5). This amounts to rescaling $\tau_{n}$.

\subsection{The Lax spectrum}

We assume $u=u^{*}$ is a $2 L$-spatially periodic stationary solution of the $n$-th $\mathrm{KdV}$ equation. The object of this section is to determine explicitly all values of $\zeta$ that result in spatially bounded, but not necessarily periodic, solutions of (3.1) and (3.7).

Definition. The Lax spectrum $\sigma_{L_{n}}$ for the $n$-th flow is the set of all $\zeta$ values such that (3.1) and (3.7) are spatially bounded.

As before, since $\zeta$ acts as the spectral parameter for the stationary Schrödinger equation, the Lax spectrum, for any of the time flows, is a subset of the real line: $\sigma_{L_{n}} \subset \mathbb{R}$.

Since $u=u^{*}$ is a stationary solution of the $n$-th $\mathrm{KdV}$ equation, $\hat{T}_{n}$ in (3.7) does not depend on $t_{n}$ and we separate variables

$$
\psi=e^{\Omega_{n} t_{n}}\left(\begin{array}{c}
\alpha_{n}(x) \\
\beta_{n}(x)
\end{array}\right)
$$

Substitution into (3.7) gives

$$
\left(\begin{array}{cc}
\hat{A}_{n}-\Omega_{n} & \hat{B}_{n} \\
\hat{C}_{n} & -\hat{A}_{n}-\Omega_{n}
\end{array}\right)\left(\begin{array}{c}
\alpha_{n} \\
\beta_{n}
\end{array}\right)=0 .
$$

Requiring a non-trivial solution of the above equation yields

$$
\Omega_{n}^{2}=\hat{A}_{n}^{2}+\hat{B}_{n} \hat{C}_{n}
$$

It is easy to show that (3.8) is independent of $x$ and any $t_{k}$ [39]. This determines a relationship between $\Omega_{n}$ and $\zeta$. In general (3.8) is an algebraic curve representation of a genus $n$ hyperelliptic Riemann surface $[7,51]$. Based on the expansion in $\zeta$ for $B_{n}(3.5)$, we see that the right-hand side of (3.8) is a polynomial in $\zeta$ of degree $2 n+1$. Furthermore, since $u^{*}$ is a (non-degenerate) genus $n$ potential it follows that all $2 n+1$ roots of the aforementioned polynomial are real and distinct [42]. Thus,

$$
\Omega_{n}^{2}=r_{n}\left(\zeta-\zeta_{1}\right) \cdots\left(\zeta-\zeta_{2 n+1}\right),
$$

for some real constants $\zeta_{1}<\cdots<\zeta_{2 n+1}$ and positive constant $r_{n}$. 
The eigenvector is given by

$$
\left(\begin{array}{c}
\alpha_{n} \\
\beta_{n}
\end{array}\right)=\gamma_{n}(x)\left(\begin{array}{c}
-\hat{B}_{n} \\
\hat{A}_{n}-\Omega_{n}
\end{array}\right),
$$

where $\gamma_{n}(x)$ is a scalar function of $x$. It is determined by substitution of the above into the first equation of the Lax pair, resulting in two linear first-order scalar differential equations for $\gamma_{n}$ which are linearly dependent. Solving gives

$$
\gamma_{n}(x)=\exp \int\left(\frac{-\partial_{x} \hat{B}_{n}-\hat{A}_{n}+\Omega_{n}}{\hat{B}_{n}}\right) d x,
$$

up to a multiplicative constant. We simplify the above. Using $\hat{A}_{n}=-\frac{1}{2} \partial_{x} \hat{B}_{n}$, we find

$$
\gamma_{n}(x)=\frac{1}{\hat{B}_{n}^{\frac{1}{2}}} \exp \left(\int \frac{\Omega_{n}}{\hat{B}_{n}} d x\right) .
$$

Each value of $\zeta$ results in two values of $\Omega_{n}$, except for the $2 n+1$ branch points $\zeta_{1}, \ldots, \zeta_{2 n+1}$ which give $\Omega_{n}=0$. Therefore, (3.9) represents two eigenvectors. This explicitly verifies the results in $[42,44,46]$, in the spectral problem for the stationary Schrödinger equation with a genus $n$ potential $\zeta$ is a double eigenvalue for all but $2 n+1$ values.

Let us determine which values of $\zeta$ result in bounded eigenfunctions (3.9):

- Consider all values of $\zeta$ for which it is possible that $\hat{B}_{n}$ (when considered as a function of $x$ ) attains zero. From (3.8), we see that this only happens for values of $\zeta$ such that $\Omega_{n}^{2}(\zeta)=\hat{A}_{n}^{2} \geq 0$, since $\hat{A}_{n} \in \mathbb{R}$ for $\zeta \in \mathbb{R}$. For the branch points $\zeta_{i}$ where $\Omega_{n}\left(\zeta_{i}\right)=0$, $i=1, \ldots, 2 n+1$, it is easily checked that the eigenfunction (3.9) is bounded, thus the zeros of $\Omega_{n}$ are part of the Lax spectrum. For all other values of $\zeta$ where $\hat{B}_{n}$ attains zero for some $x$, but $\Omega_{n}(\zeta) \neq 0$, both solutions obtained from (3.9) are unbounded, thus these $\zeta$ values are not part of the Lax spectrum.

- Consider all values of $\zeta, \Omega_{n}(\zeta) \neq 0$, such that $\hat{B}_{n}$, when considered as a function of $x$, never attains zero. From our previous considerations, we know this is true for (at least) values of $\zeta$ where $\Omega_{n}^{2}(\zeta)<0$. There is an exponential contribution from $\gamma_{n}(x)$

$$
\exp \left(\int \frac{\Omega_{n}}{\hat{B}_{n}} d x\right)
$$

which needs to be bounded. To this end, it is necessary and sufficient that

$$
\left\langle\operatorname{Re}\left(\frac{\Omega_{n}}{\hat{B}_{n}}\right)\right\rangle=0
$$

where $\langle\cdot\rangle=\frac{1}{2 L} \int_{-L}^{L} \cdot d x$ denotes the average over one period of $u^{*}$. This average is well defined since the denominator $\hat{B}_{n}$ is never zero by assumption. It follows from (3.8) that $\Omega_{n}$ is either purely real or purely imaginary. We also have $\hat{B}_{n} \in \mathbb{R}$ for $\zeta \in \mathbb{R}$. The above condition reduces to 


$$
\left\langle\frac{1}{\hat{B}_{n}}\right\rangle \operatorname{Re}\left(\Omega_{n}\right)=0
$$

We see that all values of $\zeta$ for which $\Omega_{n}$ is imaginary are part of the Lax spectrum. Now, suppose $\zeta \in \mathbb{R}$ is such that $\Omega_{n}$ is real and non-zero. Then the average term in (3.12) must be identically zero. However, since $\frac{1}{\hat{B}_{n}}$ is never zero it follows that it cannot have zero average. Therefore $\operatorname{Re}\left(\Omega_{n}\right)$ must be zero.

We conclude that the Lax spectrum consists of all $\zeta$ values for which $\Omega_{n}^{2} \leq 0$ :

$$
\sigma_{L_{n}}=\left(-\infty, \zeta_{1}\right] \cup\left[\zeta_{2}, \zeta_{3}\right] \cup \cdots \cup\left[\zeta_{2 n}, \zeta_{2 n+1}\right]
$$

This is a well-known result, but it has been deduced here in more explicit terms. Furthermore, for all values of $\zeta \in \sigma_{L_{n}}$ :

$$
\Omega_{n} \in i \mathbb{R} .
$$

In fact, $\Omega_{n}^{2}$ takes on all negative values for $\zeta \in\left(-\infty, \zeta_{1}\right]$, implying that $\Omega_{n}= \pm \sqrt{\left|\Omega_{n}^{2}\right|}$ covers the imaginary axis. Furthermore, for $\zeta \in\left[\zeta_{2}, \zeta_{3}\right], \Omega_{n}^{2}$ takes on all negative values in $\left[\Omega_{n}^{2}\left(\zeta_{2}^{*}\right), 0\right]$ twice, where $\Omega_{n}^{2}\left(\zeta_{2}^{*}\right)$ is the minimal value of $\Omega_{n}^{2}$ attained for $\zeta \in\left[\zeta_{2}, \zeta_{3}\right]$. Upon taking square roots, this implies that the interval on the imaginary axis $\left[-i \sqrt{\left|\Omega_{n}^{2}\left(\zeta_{2}^{*}\right)\right|}, i \sqrt{\left|\Omega_{n}^{2}\left(\zeta_{2}^{*}\right)\right|}\right]$ is double covered in addition to the single covering already mentioned. Similarly, for $\zeta \in\left[\zeta_{2 i}, \zeta_{2 i+1}\right], i=1, \ldots, n, \Omega_{n}^{2}$ takes on all negative values in $\left[\Omega_{n}^{2}\left(\zeta_{2 i}^{*}\right), 0\right]$ twice, where $\Omega_{n}^{2}\left(\zeta_{2 i}^{*}\right)$ is the minimal value of $\Omega_{n}^{2}$ attained for $\zeta \in\left[\zeta_{2 i}, \zeta_{2 i+1}\right]$. Symbolically, we write [12]

$$
\Omega_{n} \in i \mathbb{R} \cup\left[-i \sqrt{\left|\Omega_{n}^{2}\left(\zeta_{2}^{*}\right)\right|}, i \sqrt{\left|\Omega_{n}^{2}\left(\zeta_{2}^{*}\right)\right|}\right]^{2} \cup \cdots \cup\left[-i \sqrt{\left|\Omega_{n}^{2}\left(\zeta_{2 n}^{*}\right)\right|}, i \sqrt{\left|\Omega_{n}^{2}\left(\zeta_{2 n}^{*}\right)\right|}\right]^{2}
$$

where the square is used to denote multiplicity, see Fig. 1.

\subsection{The squared eigenfunction connection and spectral stability}

It is well known $[2,12,42]$ that the product

$$
w\left(x, t_{n}\right)=\psi_{1}\left(x, t_{n}\right) \psi_{2}\left(x, t_{n}\right)=\frac{1}{2} \partial_{x} \psi_{1}^{2}\left(x, t_{n}\right)
$$

satisfies the linear stability problem (2.14). This is known as the squared eigenfunction connection. Using the results of the previous section, we see that for stationary solutions of the $n$-th $\mathrm{KdV}$ equation the above takes the form

$$
w\left(x, t_{n}\right)=e^{2 \Omega_{n} t_{n}} \alpha_{n}(x) \beta_{n}(x)=\frac{1}{2} e^{2 \Omega_{n} t_{n}} \partial_{x} \alpha_{n}^{2}(x),
$$

where $\alpha_{n}, \beta_{n}$ are defined in (3.9). Comparing with (2.15), we immediately conclude that

$$
\lambda_{n}=2 \Omega_{n}, \quad W(x)=\alpha_{n}(x) \beta_{n}(x)=\frac{1}{2} \partial_{x} \alpha_{n}^{2}(x),
$$




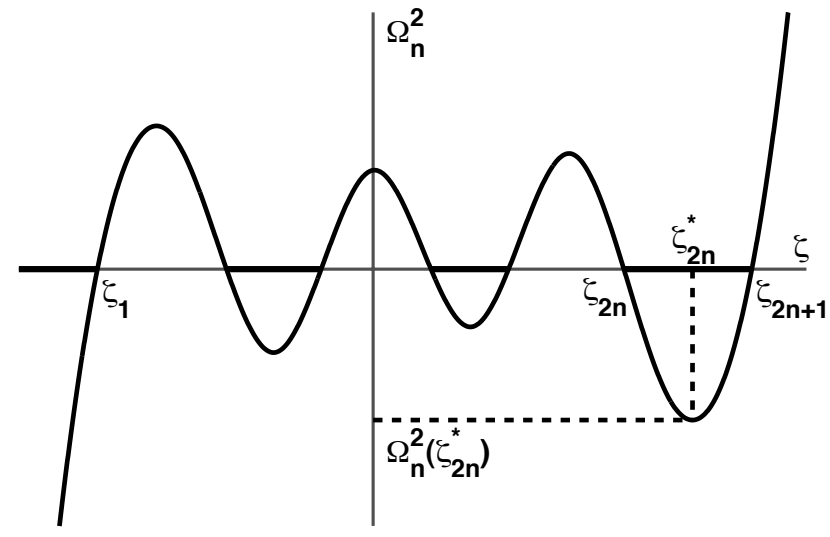

(a)

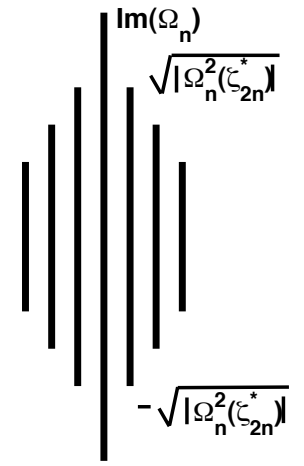

(b)

Figure 1: (a) $\Omega_{n}^{2}$ as a function of real $\zeta$. The union of the bold line segments is the Lax spectrum. (b) Corresponding plot of $\Omega_{n}$ (restricted to the Lax spectrum) in the complex plane. The vertical lines represent the multiple coverings in (3.14), which actually lie on the imaginary axis.

for all solutions obtained through the squared eigenfunction connection.

If we show that all solutions with $\operatorname{Re}\left(\lambda_{n}\right)>0$ are unbounded in $x$, then spectral stability follows. To this end, let us examine which solutions of the linear stability problem are obtained through the squared eigenfunction connection. For any given $\lambda_{n} \in \mathbb{C},(2.16)$ is a $(2 n+1)$-order differential equation. Thus, it has $2 n+1$ linearly independent solutions.

Theorem. All spatially bounded solutions of the spectral problem (2.16) with $\lambda_{n} \neq 0$ are obtained through the squared eigenfunction connection (3.15). If $\lambda_{n}=0$, then exactly $n$ linearly independent spatially bounded solutions are obtained through (3.15).

Proof. First, let us count how many solutions are obtained from the squared eigenfunction connection for a fixed value of $\lambda_{n} \neq 0$. Exactly one value of $\Omega_{n}$ is obtained through $\Omega_{n}=\lambda_{n} / 2$. Excluding the values of $\lambda_{n}$ for which the discriminant of (3.8) as a function of $\zeta$ is zero, (3.8) gives rise to $2 n+1$ values of $\zeta$. Before we consider the excluded values separately, we need to show that the $2 n+1$ functions $W(x)$ obtained as described are linearly independent.

From our previous calculations

$$
W=\alpha_{n} \beta_{n}=\left(\Omega_{n}-\hat{A}_{n}\right) \exp \left(\int \frac{2 \Omega_{n}}{\hat{B}_{n}} d x\right) .
$$

Therefore, as long as there is an exponential contribution, the $2 n+1$ solutions $W$ corresponding to the $2 n+1$ values of $\zeta$ are linearly independent: indeed for different $\zeta$ different terms with singularities of different order in the complex $x$-plane are present with different coefficients. From the above we see that there is an exponential contribution if and only if $\Omega_{n} \neq 0$, which is true since $\Omega_{n}=\lambda_{n} / 2 \neq 0$ by assumption. Furthermore, if $\operatorname{Re}\left(\lambda_{n}\right)>0$ it follows that $\operatorname{Re}\left(\Omega_{n}\right)>0$. This implies $\zeta$ is not in the Lax spectrum. Therefore, all $2 n+1$ solutions obtained from the squared eigenfunction connection corresponding to $\operatorname{Re}\left(\lambda_{n}\right)>0$ are unbounded in $x$.

For the values of $\lambda_{n}$ at which the discriminant of (3.8) as a function of $\zeta$ is zero, only $2 n$ solutions are obtained (see note below). The other solution can be obtained through reduction of order. This 
introduces algebraic growth, therefore it is unbounded in $x$. We have thus shown that all bounded solutions corresponding to $\lambda_{n} \neq 0$ are obtained through the squared eigenfunction connection.

Note: Extra care must be taken if the degeneracy is stronger, such as two local minima of $\Omega_{n}^{2}$ being equal when the discriminant of (3.8) as a function of $\zeta$ is zero. In such cases less than $2 n$ solutions are obtained. However, a simple perturbation argument resolves these higher degeneracies and unbounded solutions result.

Now, assume $\lambda_{n}=0$. It follows that $\Omega_{n}=\lambda_{n} / 2=0$. Substituting $\Omega_{n}=0$ into (3.16) gives

$$
W=-\hat{A}_{n} .
$$

Note that $\hat{A}_{n}$ is linearly related to the $A_{j}$ from the $\tau_{j}$-hierarchy. Using that $A_{n}=-\frac{1}{2} \partial_{x} B_{n},(3.6)$, and the expansion $A_{n}=\sum_{j=0}^{n} a_{j}(x) \zeta^{j}$ we find

$$
a_{j-1}=\frac{1}{8}\left(-\partial_{x}^{2} a_{j}-\frac{2}{3} u a_{j}-\frac{1}{3} u_{x} \partial_{x}^{-1} a_{j}\right) .
$$

The above is precisely the recursion operator (2.6) which generates the KdV hierarchy, i.e.,

$$
a_{j-1}=\frac{1}{8} \mathcal{R} a_{j}
$$

Using $a_{n}=0, a_{n-1}=\frac{1}{24} b_{n} u_{x}=\frac{1}{24} b_{n} u_{\tau_{0}}$ gives

$$
a_{j-1}=\frac{1}{8} \mathcal{R} a_{j}=d_{j} u_{\tau_{n-j}}, \quad j=n-1, \ldots, 1,
$$

for some constants $d_{j}$. In other words, each $a_{j}(x)$ is a linear multiple of $u_{\tau_{n-j}}, j=1, \ldots, n$. Since $\hat{A}_{n}$ is a linear combination of all the lower-order flows, the above result gives that $\hat{A}_{n}$ can be expressed as a linear combination of the $u_{t_{n-j}}$. Therefore, for $\lambda_{n}=0$ we obtain $n$ linearly independent solutions $u_{t_{n-j}}^{*}, j=1, \ldots, n$, from the squared eigenfunction connection. Of course, each $u_{t_{n-j}}^{*}$, $j=1, \ldots, n$, is expressed in terms of $u^{*}$ and its $x$-derivatives through the KdV hierarchy (2.7).

As seen in this proof, there is no stability spectrum with $\operatorname{Re}\left(\lambda_{n}\right)>0$. Therefore, we immediately conclude $t_{n}$-spectral stability. We summarize the above results:

Theorem (Spectral stability). All periodic genus $n$ solutions of the KdV equation are $t_{n^{-}}$ spectrally stable with respect to spatially bounded perturbations. The spectrum of their associated linear stability problem $(2.16)$ is explicitly given by $\sigma\left(J \mathcal{L}_{n}\right)=i \mathbb{R}$, or, accounting for multiplicities,

$$
\sigma\left(J \mathcal{L}_{n}\right)=i \mathbb{R} \cup\left[-2 i \sqrt{\left|\Omega_{n}^{2}\left(\zeta_{2}^{*}\right)\right|}, 2 i \sqrt{\left|\Omega_{n}^{2}\left(\zeta_{2}^{*}\right)\right|}\right]^{2} \cup \cdots \cup\left[-2 i \sqrt{\left|\Omega_{n}^{2}\left(\zeta_{2 n}^{*}\right)\right|}, 2 i \sqrt{\left|\Omega_{n}^{2}\left(\zeta_{2 n}^{*}\right)\right|}\right]^{2}
$$

\section{Nonlinear stability}

With spectral stability established, let us revisit the nonlinear stability theorem as applied to our problem. We have the following: 
- It is an application of the SCS basis lemma in $[16,28]$ that the eigenfunctions $W$ form a basis for $L_{\text {per }}^{2}([-N L, N L])$, for any integer $N$, when the potential $u$ is periodic in $x$ with period $2 L$.

- As seen in the proof of spectral stability, the infinitesimal generators of $G$ :

$$
\partial_{t_{0}}, \partial_{t_{1}}, \ldots, \partial_{t_{n-1}}
$$

acting on the solution $u^{*}$ are in the kernel of $\hat{H}_{n}^{\prime \prime}\left(u^{*}\right)$. In fact, when restricted to the space $\mathbb{V}_{0}$, the infinitesimal generators of $G$ span the kernel of $\hat{H}_{n}^{\prime \prime}\left(u^{*}\right)[16,27]$. As we see below, the kernel of $\hat{H}_{m}^{\prime \prime}\left(u^{*}\right)$ is equal to that of $\hat{H}_{n}^{\prime \prime}\left(u^{*}\right)$ for any $m \geq n$.

What is left to verify is the last condition in the nonlinear stability theorem, i.e., we need to find an $m$ such that

$$
K_{m}(W)=\left\langle W, \hat{H}_{m}^{\prime \prime}\left(u^{*}\right) W\right\rangle=\int_{-N L}^{N L} \bar{W}(x) \mathcal{L}_{m} W(x) d x \geq 0
$$

for all $W \in \mathbb{V}_{0}$ with equality obtained only for $W$ in the kernel of $\mathcal{L}_{m}=\hat{H}_{m}^{\prime \prime}\left(u^{*}\right)$.

Assume $m>n$ (what follows is trivial for $m=n$ ) and that $u^{*}$ is a stationary solution of the $n$-th flow. It is a stationary solution of the $m$-th flow as well, for some choice of the constants $c_{m, 0}, \ldots, c_{m, n-1}$. Now, consider the time component of the Lax hierarchy for the $m$-th flow,

$$
\psi_{t_{m}}=\hat{T}_{m} \psi
$$

Proceeding as we did for the $n$-th flow, we look for solutions of the form

$$
\psi\left(x, t_{m}\right)=e^{\Omega_{m} t_{m}} \tilde{\psi}(x),
$$

where due to the commuting properties of the flows, the Lax equations for $i \geq n$ share the same complete set of eigenfunctions ${ }^{3}$

$$
\tilde{\psi}(x)=\left(\begin{array}{c}
\alpha_{n} \\
\beta_{n}
\end{array}\right) .
$$

For $i<n$ we can no longer separate variables. As before, this determines a relationship between $\Omega_{m}$ and $\zeta$, and in general $\Omega_{m}^{2}$ defines an algebraic curve corresponding to a genus $m$ Riemann surface. However, when evaluated at a stationary solution of the $n$-th flow this curve is singular, and corresponds to a genus $n$ surface.

Theorem. Let $u^{*}$ be a stationary solution of the $n$-th $\mathrm{KdV}$ equation. Then for all $m>n$, the $m$-th eigenvalue equation reduces to

$$
\Omega_{m}^{2}(\zeta)=p_{m}^{2}(\zeta) \Omega_{n}^{2}(\zeta)
$$

\footnotetext{
${ }^{3}$ There are two different complete sets of eigenfunctions. The solutions of the Lax problem $\tilde{\psi}(x)$ are complete since they are the eigenfunctions of a Sturm-Liouville problem. The completeness of the eigenfunctions of the linear stability problem $W(x)$ follows from the SCS basis lemma.
} 
where $p_{m}(\zeta)$ is a polynomial of degree $m-n$ in $\zeta$. Furthermore, $p_{m}(\zeta)$ depends on the free parameters $c_{m, n}, \ldots, c_{m, m-1}$ such that $c_{m, j}$ appears in the coefficients of $\zeta^{j-n}$ and lower. Therefore, the free parameters $c_{m, n}, \ldots, c_{m, m-1}$ give us total control over the roots of $p_{m}(\zeta)$.

Proof. For $m>n$, we impose $\hat{H}_{m}^{\prime}\left(u^{*}\right)=0$. Without loss of generality, we assume that the free constants are determined in such a way that for all $m>n$ the $m$-th Hamiltonian takes the form

$$
\hat{H}_{m}(u)=\tilde{H}_{m}(u)+\tilde{c}_{m, m-1} \hat{H}_{m-1}(u)+\cdots+\tilde{c}_{m, n} \hat{H}_{n}(u),
$$

with $\tilde{H}_{m}^{\prime}\left(u^{*}\right)=0$. Each constant $\tilde{c}_{m, j}$ is expressed in terms of the constants $c_{m, k}, k \geq j$. This is not necessary in practice, it only makes the proof more transparent. In this case, when evaluated at $u^{*}$ all of the higher-order flows $u_{t_{j}}, j>n$, become linearly dependent. Hence, each $\hat{T}_{j}, j>n$, becomes a linear multiple of $\hat{T}_{n}$. Thus,

$$
\begin{aligned}
\hat{T}_{m} & =\tilde{p}_{m}(\zeta) \hat{T}_{n}+\tilde{c}_{m, m-1} \tilde{p}_{m-1}(\zeta) \hat{T}_{n}+\cdots+\tilde{c}_{m, n} \tilde{p}_{n}(\zeta) \hat{T}_{n} \\
& =p_{m}(\zeta) \hat{T}_{n}
\end{aligned}
$$

where $p_{m}(\zeta)=\tilde{p}_{m}(\zeta)+\tilde{c}_{m, m-1} \tilde{p}_{m-1}(\zeta)+\cdots+\tilde{c}_{m, n} \tilde{p}_{n}(\zeta)$, and each polynomial $\tilde{p}_{j}(\zeta)$ is of degree $j-n$ in $\zeta$. The existence of nontrivial solutions of the eigenvalue problem imposes

$$
0=\operatorname{det}\left(\hat{T}_{m}-\Omega_{m} I\right)=\operatorname{det}\left(p_{m}(\zeta) \hat{T}_{n}-\Omega_{m} I\right) .
$$

Therefore, $\Omega_{m}^{2}=p_{m}^{2}(\zeta) \Omega_{n}^{2}$. Expressing $\tilde{c}_{m, j}$ in terms of $c_{m, j}$ completes the proof.

From the squared eigenfunction connection we have

$$
2 \Omega_{m} W(x)=J \mathcal{L}_{m} W(x) .
$$

Recall that on $\mathbb{V}_{0}$, the operator $J$ is invertible so that

$$
\mathcal{L}_{m} W(x)=2 \Omega_{m} J^{-1} W(x) .
$$

Therefore,

$$
K_{m}(\zeta)=\int_{-N L}^{N L} \bar{W} \mathcal{L}_{m} W d x=2 \Omega_{m}(\zeta) \int_{-N L}^{N L} \bar{W} J^{-1} W d x=\frac{\Omega_{m}(\zeta)}{\Omega_{n}(\zeta)} K_{n}(\zeta),
$$

where $W$, and hence $K_{n}$, is parameterized in terms of the Lax parameter $\zeta$. From the previous theorem, all values of $\zeta$ for which $\Omega_{n}=0$ also give $\Omega_{m}=0$. Thus, these values of $\zeta$ pose no problem in (4.1). Substituting for $\Omega_{m}$ gives

$$
K_{m}(\zeta)=p_{m}(\zeta) K_{n}(\zeta)
$$

Thus, when considering stationary solutions of the $n$-th flow, one simply needs to calculate $K_{n}$ in order to calculate any of the higher-order $K_{i}$. Let us do so. We have

$$
W(x)=\alpha_{n}(x) \beta_{n}(x)=\frac{1}{2} \partial_{x} \alpha_{n}^{2}(x) .
$$

Therefore, the integrand in $K_{n}$ is given by 


$$
\bar{W} \mathcal{L}_{n} W=\bar{\alpha}_{n} \bar{\beta}_{n} \Omega_{n} \alpha_{n}^{2}=\Omega_{n}\left|\alpha_{n}\right|^{2} \bar{\beta}_{n} \alpha_{n}
$$

On the Lax spectrum $\left|\gamma_{n}\right|^{2}=1 / \hat{B}_{n}$, since the exponent in (3.10) is imaginary. This gives

$$
\left|\alpha_{n}\right|^{2}=\left|\gamma_{n}\right|^{2} \hat{B}_{n}^{2}=\hat{B}_{n}
$$

and

$$
\bar{\beta}_{n} \alpha_{n}=\left|\gamma_{n}\right|^{2} \overline{\left(\hat{A}_{n}-\Omega_{n}\right)}\left(-\hat{B}_{n}\right)=-\overline{\left(\hat{A}_{n}-\Omega_{n}\right)}=-\left(\hat{A}_{n}+\Omega_{n}\right),
$$

where, again, we used that $\hat{A}_{n}$ is real and $\Omega_{n}$ is imaginary on the Lax spectrum. This gives

$$
\bar{W} \mathcal{L}_{n} W=-\Omega_{n} \hat{B}_{n}\left(\hat{A}_{n}+\Omega_{n}\right) .
$$

Therefore

$$
K_{n}(\zeta)=-\Omega_{n} \int_{-N L}^{N L} \hat{B}_{n} \hat{A}_{n} d x-\Omega_{n}^{2} \int_{-N L}^{N L} \hat{B}_{n} d x .
$$

Using that $\hat{B}_{n} \hat{A}_{n}$ is periodic and a total derivative gives the final result

$$
K_{n}(\zeta)=-\Omega_{n}^{2} \int_{-N L}^{N L} \hat{B}_{n} d x
$$

Note that (4.2) is valid only on the Lax spectrum. However, we find it convenient to formally consider (4.2) as defining $K_{n}$ as a function of all real $\zeta$ in our considerations below. This poses no problems in the application of the orbital stability theorem, as it is only concerned with the sign of $K_{n}$ when evaluated at $\zeta \in \sigma_{L_{n}}$.

Consider the sign of $K_{n}(\zeta)$ on the Lax spectrum $\sigma_{L_{n}}$ :

- Since $u^{*}$ is $t_{n}$-spectrally stable, $\Omega_{n}^{2} \leq 0$ on $\sigma_{L_{n}}$. Therefore, we need to consider the sign of the integral term $\int_{-N L}^{N L} \hat{B}_{n} d x$ in (4.2).

- The Lax spectrum $\sigma_{L_{n}}$ has $n+1$ components: $\left(-\infty, \zeta_{1}\right],\left[\zeta_{2}, \zeta_{3}\right], \ldots,\left[\zeta_{2 n}, \zeta_{2 n+1}\right]$. Previously, we saw that $\hat{B}_{n}$ never attains zero (as a function of $x$ ) for $\zeta \in \sigma_{L_{n}}$, except possibly at the endpoints of these components where $\Omega_{n}(\zeta)=0$. This implies that the integral term $\int_{-N L}^{N L} \hat{B}_{n} d x$ in (4.2) is not zero and has fixed sign on each component of the Lax spectrum. However, that sign may change from one component to the next. Therefore, $K_{n}(\zeta)$ can change sign only in the gaps where $\zeta \notin \sigma_{L_{n}}$ or at the band edges where $\Omega_{n}(\zeta)=0$.

Thus, $K_{n}(\zeta)$ is not guaranteed to have fixed sign on the entire Lax spectrum, but only on each component separately. Hence, no stability conclusions can be drawn from $K_{n}(\zeta)$. However, considering $K_{2 n}(\zeta)$ introduces the requisite number of constants to allow us to make $K_{2 n}(\zeta)$ of definite sign on the entire Lax spectrum. We have

$$
K_{2 n}(\zeta)=p_{2 n}(\zeta) K_{n}(\zeta)
$$

where $p_{2 n}(\zeta)$ is a polynomial in $\zeta$ of degree $2 n-n=n$. Since we have total control over the roots of $p_{2 n}(\zeta)$, we choose the $n$ constants $c_{2 n, n}, \ldots, c_{2 n, 2 n-1}$ so that $p_{2 n}(\zeta)$ changes sign whenever the 


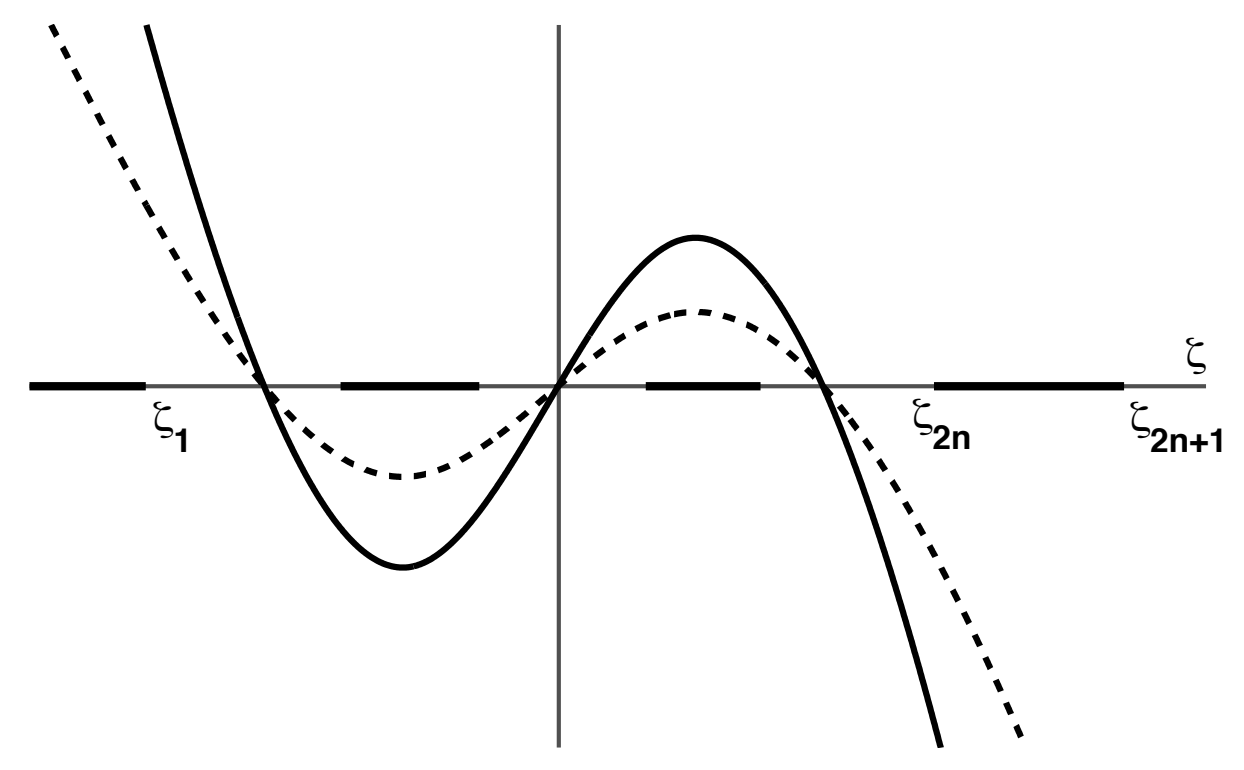

Figure 2: The bold and dashed curves represent the integral term $\int_{-N L}^{N L} \hat{B}_{n} d x$ in $K_{n}(\zeta)$ and $p_{2 n}(\zeta)$ respectively, considered as functions of real $\zeta$. The union of the thick line segments on the real axis is the Lax spectrum $\sigma_{L_{n}}$. Both curves have different signs on various components of the Lax spectrum, but their product has fixed sign on the entire Lax spectrum.

integral term in $K_{n}(\zeta)$ changes sign, see Fig. 2. This is always possible since the integral term in $K_{n}(\zeta)$ is a polynomial in $\zeta$ of degree $n$. This makes $K_{2 n}(\zeta)$ of definite sign on the entire Lax spectrum, establishing the last condition in the nonlinear stability theorem.

We have proved the following:

Theorem (Orbital stability). Spatially periodic genus $n$ solutions of the KdV equation are orbitally stable (under the time dynamics of any of the KdV equations) with respect to perturbations in

$$
\mathbb{V}_{0}=\left\{v \in H_{p e r}^{2 n+1}([-N L, N L]): \frac{1}{2 N L} \int_{-N L}^{N L} v d x=0\right\}
$$

where $2 L$ is the period of the initial condition and $N$ is any positive integer.

\section{Remarks.}

- There is no restriction on the spatial average of the finite-genus solution, only on the spatial average of the perturbation.

- The choice of constants, $c_{2 n, n}, \ldots, c_{2 n, 2 n-1}$, that makes $K_{2 n}(\zeta)$ of definite sign is not unique. For example, one could require $p_{2 n}(\zeta)$ to have the same $n$ zeros as the integral term in $K_{n}(\zeta)$. One could instead require $p_{2 n}(\zeta)$ to have $\zeta_{2 j}$ (or $\zeta_{2 j-1}$ ) as a zero if $K_{n}(\zeta)$ has an undesired sign on the band $\left[\zeta_{2 j}, \zeta_{2 j+1}\right] \subset \sigma_{L_{n}}$.

- In the soliton limit the allowed bands in (3.13) collapse to single points [22]. Thus, the operator $\hat{H}_{n}^{\prime \prime}\left(u^{*}\right)$ may have up to $n$ unstable directions and the theory of [43] applies. It is interesting 
to note that our formulation using $\hat{H}_{2 n}^{\prime \prime}\left(u^{*}\right)$ eliminates the extra machinery (Theorem 2 of [26]) required to negotiate the unstable directions, and orbital stability follows from Theorem 1 of [26]. To turn this into a formal proof for the soliton case, one needs to examine the interplay between the infinite period limit and the steps necessary for our method.

- The infinitesimal generators of $G$ also span the tangent space of the Abelian torus on which the Riemann theta function in (1.4) is defined [46], as they are linearly related to $\partial_{\phi_{01}}, \ldots, \partial_{\phi_{0 n}}$. In terms of a Hamiltonian description with action-angle variables, these generators give the infinitesimal version of the motion of the angles on the Liouville-Arnold torus (directly related to the Abelian torus underlying (1.4)) of the corresponding integrable dynamics [20].

\section{Examples}

\subsection{Genus 1: the cnoidal wave}

We repeat the results of $[12,16]$ as an illustration of our general framework applied to the genus one case. Consider the genus one example (2.11)

with period $2 L=2 \mathrm{~K}(k)(2.10)$.

$$
u^{*}=12 k^{2} \mathrm{cn}^{2}(x, k),
$$

We have

$$
\hat{T}_{1}=T_{1}+c_{1,0} T_{0}
$$

where as before $c_{1,0}=8 k^{2}-4$. From

$$
\operatorname{det}\left(\hat{T}_{1}-\Omega_{1} I\right)=0
$$

a direct calculation gives

$$
\Omega_{1}^{2}=16\left(\zeta-\zeta_{1}\right)\left(\zeta-\zeta_{2}\right)\left(\zeta-\zeta_{3}\right)
$$

with

$$
\zeta_{1}=k^{2}-1<\zeta_{2}=2 k^{2}-1<\zeta_{3}=k^{2},
$$

for $k \in(0,1)$. The Lax spectrum is

$$
\sigma_{L_{1}}=\left(-\infty, k^{2}-1\right] \cup\left[2 k^{2}-1, k^{2}\right] .
$$

To examine orbital stability, let us calculate $K_{1}$. We have

$$
\begin{aligned}
K_{1}(\zeta) & =-\Omega_{1}^{2} \int_{-N L}^{N L} \hat{B}_{1} d x \\
& =-\Omega_{1}^{2} \int_{-N L}^{N L}\left(-4 \zeta-\frac{1}{3} u^{*}+c_{1,0}\right) d x \\
& =-\Omega_{1}^{2} \int_{-N L}^{N L}\left(-4 \zeta-4 k^{2} \operatorname{cn}^{2}(x, k)+8 k^{2}-4\right) d x
\end{aligned}
$$


There are two components to the Lax spectrum. We see that $K_{1}(\zeta) \geq 0$ on the first component $\zeta \in\left(-\infty, k^{2}-1\right]$, and $K_{1}(\zeta) \leq 0$ on the second component $\zeta \in\left[2 k^{2}-1, k^{2}\right]$. In both cases equality is obtained only at the endpoitns, where $\Omega_{1}(\zeta)=0$. Therefore, no stability conclusions can be drawn from $K_{1}(\zeta)$.

Let us calculate $K_{2}(\zeta)$. We have

$$
\hat{T}_{2}=T_{2}+c_{2,1} T_{1}+c_{2,0} T_{0},
$$

where as before $c_{2,0}=c_{2,1}\left(8 k^{2}-4\right)-56 k^{4}+56 k^{2}-16$ and $c_{2,1}$ is free. From

$$
\operatorname{det}\left(\hat{T}_{2}-\Omega_{2} I\right)=0
$$

another direct calculation gives

$$
\Omega_{2}^{2}=16\left(8 k^{2}-4+4 \zeta-c_{2,1}\right)^{2}\left(\zeta-\zeta_{1}\right)\left(\zeta-\zeta_{2}\right)\left(\zeta-\zeta_{3}\right)=\left(4 \zeta+8 k^{2}-4-c_{2,1}\right)^{2} \Omega_{1}^{2} .
$$

We choose $c_{2,1}$ such that $4 \zeta+8 k^{2}-4-c_{2,1}$ has the same zero as the integrand of $K_{1}(\zeta)$. This choice of $c_{2,1}$ makes $K_{2}(\zeta)$ of definite sign on the Lax spectrum, and verifies orbital stability.

In fact, $4 \zeta+8 k^{2}-4-c_{2,1}$ is zero when $\zeta=1-2 k^{2}+c_{2,1} / 4$. Imposing that this sign change takes place in the gap (or on one of its edges) gives

$$
1-2 k^{2}+c_{2,1} / 4 \geq k^{2}-1
$$

and

$$
1-2 k^{2}+c_{2,1} / 4 \leq 2 k^{2}-1
$$

This results in

$$
4\left(3 k^{2}-2\right) \leq c_{2,1} \leq 4\left(4 k^{2}-2\right),
$$

which has an entire interval of solutions for all values of the elliptic modulus $k \in(0,1)$. Any choice of $c_{2,1}$ satisfying the above constraint makes $K_{2}(\zeta)$ of definite sign on the Lax spectrum, and

$$
\hat{H}_{2}=H_{2}+c_{2,1} H_{1}+c_{2,0} H_{0}
$$

serves as a Lyapunov function.

Though not necessary for stability, let us calculate $\Omega_{3}$ for illustrative purposes. We have

$$
\hat{T}_{3}=T_{3}+c_{3,2} T_{2}+c_{3,1} T_{1}+c_{3,0} T_{0} .
$$

Imposing $u^{*}$ is stationary gives

$$
c_{3,0}=c_{3,2}\left(-56 k^{4}+56 k^{2}-16\right)+c_{3,1}\left(8 k^{2}-4\right)+384 k^{6}-576 k^{4}+320 k^{2}-64,
$$

where $c_{3,2}$ and $c_{3,1}$ are free parameters. From

$$
\operatorname{det}\left(\hat{T}_{3}-\Omega_{3} I\right)=0
$$

another direct calculation gives 


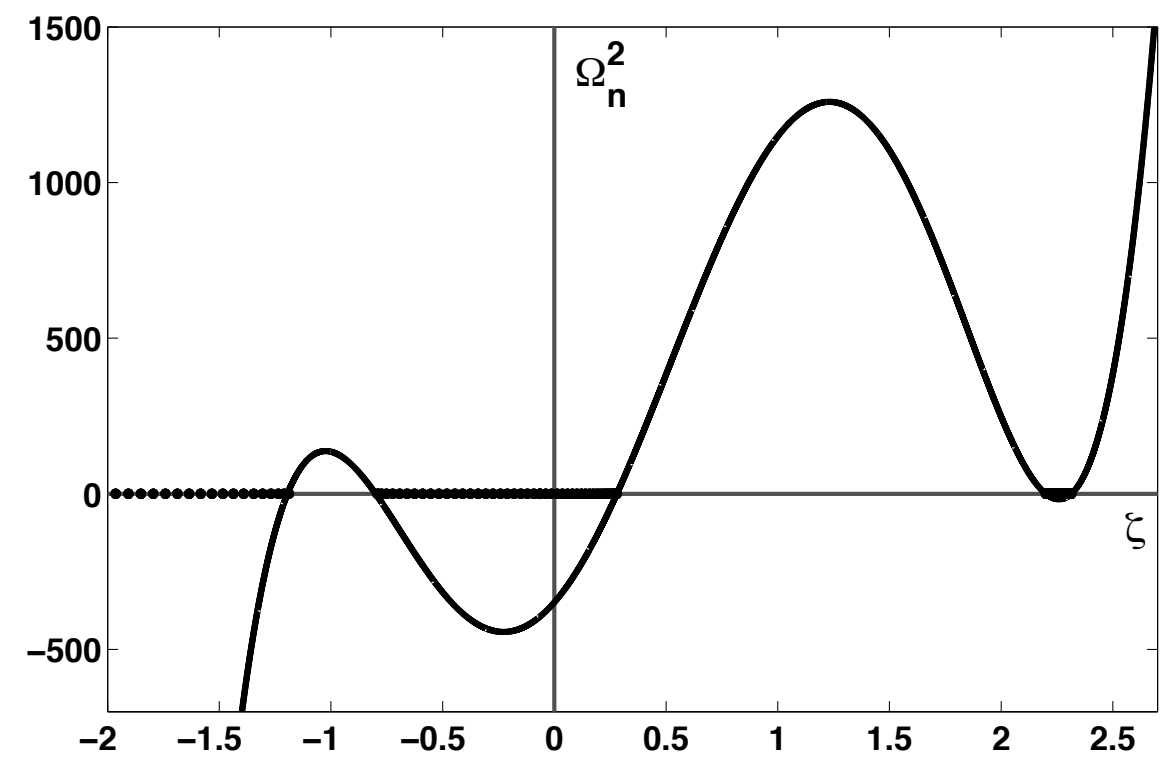

Figure 3: $\Omega_{2}^{2}$ as a function of real $\zeta$ for the Dubrovin-Novikov solution with $k=0.8$ in (5.1). The union of the dotted line segments on the horizontal axis is the numerically computed Lax spectrum using Hill's method with 81 Fourier modes and 49 different Floquet exponents, see $[18,17]$.

$$
\Omega_{3}^{2}=\left(16 \zeta^{2}+\left(32 k^{2}-16-4 c_{3,2}\right) \zeta+56 k^{4}-56 k^{2}-8 k^{2} c_{3,2}+4 c_{3,2}+16+c_{3,1}\right)^{2} \Omega_{1}^{2} .
$$

Now, $c_{3,2}$ allows us to choose the coefficient of $\zeta$ and $c_{3,1}$ allows us to choose the constant term. Hence, we have total control over the roots of the outside polynomial.

\subsection{Genus 2: the Dubrovin-Novikov solution}

We consider the genus two Lamé-Ince potential [19, 22, 31, 32]

$$
u^{*}=-36 \wp\left(x, g_{2}, g_{3}\right),
$$

where $\wp\left(\cdot, g_{2}, g_{3}\right)$ is the Weierstrass elliptic function with invariants $g_{2}$ and $g_{3}$ [41]. A direct substitution shows that $u^{*}$ is not a stationary solution of the first KdV equation for any choice of the constant $c_{1,0}$. In fact, the solution $u(x, t)$ evolving from $u^{*}$ takes the form

$$
u(x, t)=-12\left(\wp\left(x-x_{1}(t), g_{2}, g_{3}\right)+\wp\left(x-x_{2}(t), g_{2}, g_{3}\right)+\wp\left(x-x_{3}(t), g_{2}, g_{3}\right)\right),
$$

where the dynamics of $x_{j}(t), j=1,2,3$, is governed by a nonlinear dynamical system [3]. Contrary to the genus one case, the solution $u(x, t)$ does not represent all periodic genus two solutions. In fact, it is considered the simplest periodic genus two solution, as noted by Dubrovin and Novikov, who integrated the $\mathrm{KdV}$ equation with $u^{*}$ as an initial condition [22, 47]. It was later shown that the Dubrovin-Novikov solution is time periodic as well [23]. We examine it because it is a solution with genus greater than one for which explicit analysis is relatively straightforward. 
Using the translation invariance of the $\mathrm{KdV}$ equation, we rewrite the above in the more convenient form $[15,41]$

$$
u^{*}=36 k^{2} \mathrm{cn}^{2}(x, k) \text {. }
$$

Imposing that $u^{*}$ is a stationary solution of the second $\mathrm{KdV}$ equation gives

$$
c_{2,0}=424 k^{4}-424 k^{2}+64, \quad c_{2,1}=40 k^{2}-20 .
$$

From

$$
\operatorname{det}\left(\hat{T}_{2}-\Omega_{2} I\right)=0
$$

we find

where

$$
\Omega_{2}^{2}=256\left(\zeta-\zeta_{1}\right)\left(\zeta-\zeta_{2}\right)\left(\zeta-\zeta_{3}\right)\left(\zeta-\zeta_{4}\right)\left(\zeta-\zeta_{5}\right)
$$

$$
\left(\zeta_{1}, \zeta_{2}, \zeta_{3}, \zeta_{4}, \zeta_{5}\right)=\left(4 k^{2}-2-2 \sqrt{k^{4}-k^{2}+1}, 5 k^{2}-4,2 k^{2}-1,5 k^{2}-1,4 k^{2}-2+2 \sqrt{k^{4}-k^{2}+1}\right) .
$$

It is easily checked that all of the above roots are real and distinct for $k \in(0,1)$. Therefore, the Lax spectrum is

$$
\sigma_{L_{2}}=\left(-\infty, \zeta_{1}\right] \cup\left[\zeta_{2}, \zeta_{3}\right] \cup\left[\zeta_{4}, \zeta_{5}\right]
$$

which is a confirmation of numerical results, see Fig. 3. Also, $\Omega_{2}$ has two bands of increasingly higher density around the origin. This confirms the numerical results for the linear stability problem, see Figs. 4 and 5.

To examine nonlinear stability, we first calculate $K_{2}(\zeta)$ :

$$
\begin{aligned}
K_{2}(\zeta) & =-\Omega_{2}^{2} \int_{-N L}^{N L} \hat{B}_{2} d x \\
& =-\Omega_{2}^{2} \int_{-N L}^{N L}\left(16 \zeta^{2}+\left(\frac{4}{3} u^{*}-4 c_{2,1}\right) \zeta+\frac{1}{3} u_{x x}^{*}+\frac{1}{6} u^{* 2}-\frac{1}{3} c_{2,1} u^{*}+c_{2,0}\right) d x
\end{aligned}
$$

As expected, the integral part of $K_{2}(\zeta)$ is a polynomial of degree two in $\zeta$. There are three components to the Lax spectrum. One can check that $K_{2}(\zeta) \geq 0$ on the first component $\zeta \in$ $\left(-\infty, \zeta_{1}\right], K_{2}(\zeta) \leq 0$ on the second component $\zeta \in\left[\zeta_{2}, \zeta_{3}\right]$, and $K_{2}(\zeta) \geq 0$ on the third component $\zeta \in\left[\zeta_{4}, \zeta_{5}\right]$. In all three cases equality is obtained only at the endpoints, where $\Omega_{2}(\zeta)=0$. Therefore, no stability conclusions can be drawn from $K_{2}(\zeta)$.

For orbital stability we need to consider $K_{4}$. Imposing that $u^{*}$ is a stationary solution of the fourth $\mathrm{KdV}$ equation results in

$$
\begin{aligned}
c_{4,0}= & \left(-16192 k^{6}+24288 k^{4}-10656 k^{2}+1280\right) c_{4,3}+\left(424 k^{4}-424 k^{2}+64\right) c_{4,2} \\
& +467616 k^{8}-935232 k^{6}+677664 k^{4}-210048 k^{2}+21504, \\
c_{4,1}= & \left(-1176 k^{4}+1176 k^{2}-336\right) c_{4,3}+\left(40 k^{2}-20\right) c_{4,2} \\
& +30848 k^{6}-46272 k^{4}+26304 k^{2}-5440,
\end{aligned}
$$




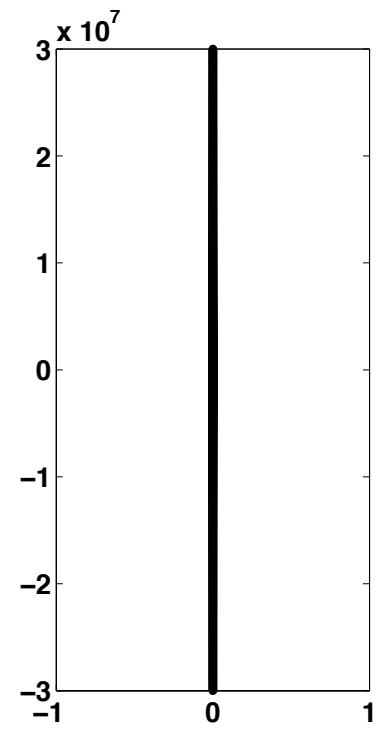

(a)

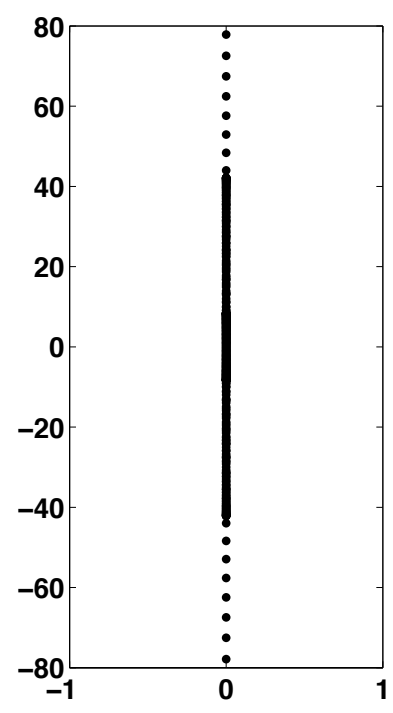

(b)

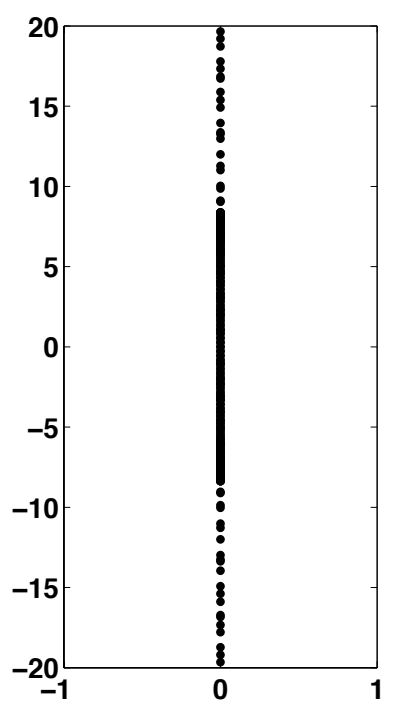

(c)

Figure 4: (a) The numerically computed spectrum for the Dubrovin-Novikov solution with $k=0.8$ using Hill's method with 81 Fourier modes and 49 different Floquet exponents, see [17, 18]; (b) A blow-up of (a) around the origin, showing a band of higher spectral density; (c) A blow-up of (b) around the origin, showing another band of even higher spectral density. The analytically predicted values for the band ends using (5.1) are $\pm 2 i \sqrt{\left|\Omega_{2}^{2}\left(\zeta_{2}^{*}\right)\right|} \approx \pm 42.14 i$ and $\pm 2 i \sqrt{\left|\Omega_{2}^{2}\left(\zeta_{4}^{*}\right)\right|} \approx \pm 8.38 i$, in agreement with the numerical results above.

where $c_{4,2}$ and $c_{4,3}$ are free parameters.

From $\operatorname{det}\left(\hat{T}_{4}-\Omega_{4} I\right)=0$ we find

$$
\Omega_{4}^{2}=p_{4}(\zeta)^{2} \Omega_{2}^{2}
$$

where

$$
p_{4}(\zeta)=16 \zeta^{2}+\left(160 k^{2}-4 c_{4,3}-80\right) \zeta+1176 k^{4}+\left(-40 c_{4,3}-1176\right) k^{2}+20 c_{4,3}+c_{4,2}+336 .
$$

Therefore, $c_{4,3}$ allows us to choose the coefficient of $\zeta$ and $c_{4,2}$ allows us to choose the constant term, giving us total control over the roots of $p_{4}(\zeta)$. Imposing that it has the same roots as the integral part of $K_{2}(\zeta)$ determines $c_{4,3}$ and $c_{4,2}$ such that $K_{4}(\zeta)$ is of definite sign, verifying orbital stability.

\section{Acknowledgements}

We thank Chris Curtis and Todd Kapitula for useful comments. Further, we gratefully acknowledge support from the National Science Foundation under grants NSF-DMS-0604546 (BD) and NSF-DMS-VIGRE-0354131 (MN). Any opinions, findings, and conclusions or recommendations 


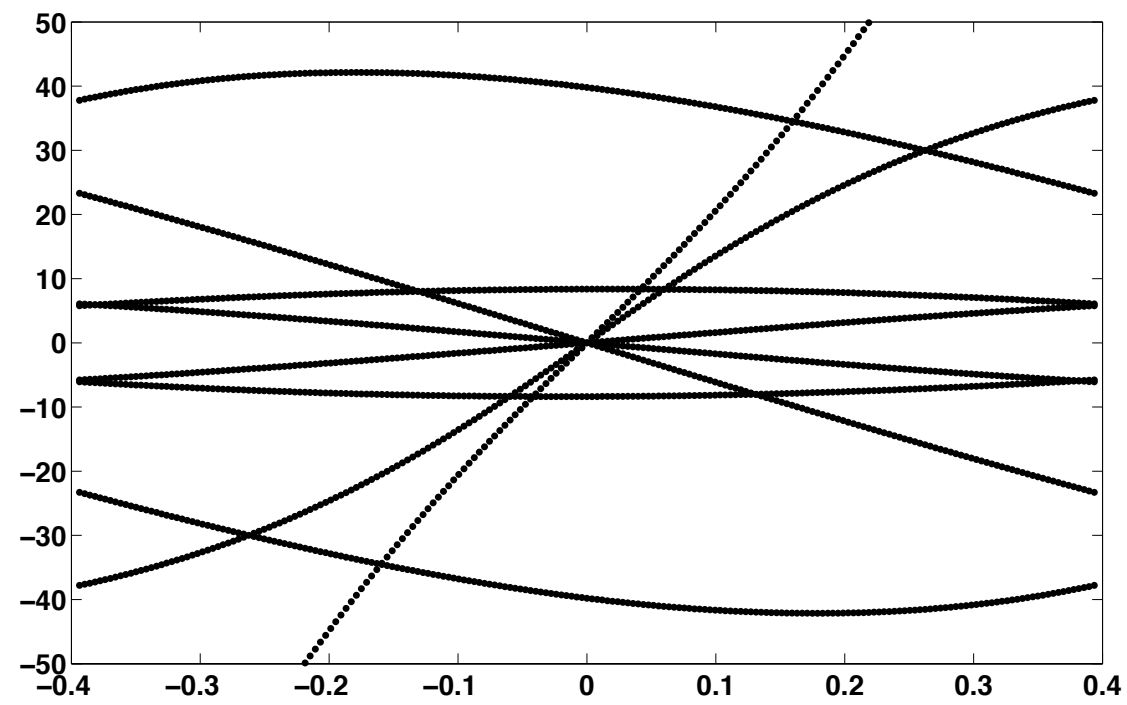

Figure 5: The imaginary part of $\lambda_{n}$ as a function of $\mu$, where $\mu$ is the Floquet parameter corresponding to a Bloch-wave decomposition of the eigenfunction $[17,18]$, demonstrating the higher spectral density around the origin of the imaginary axis.

expressed in this material are those of the authors and do not necessarily reflect the views of the funding sources.

\section{References}

[1] M. J. Ablowitz, D. J. Kaup, A. C. Newell, and H. Segur. The inverse scattering transformFourier analysis for nonlinear problems. Studies in Appl. Math., 53:249-315, 1974.

[2] M. J. Ablowitz and H. Segur. Solitons and the inverse scattering transform. SIAM, Philadelphia, PA, 1981.

[3] H. Airault, H. P. McKean, and J. Moser. Rational and elliptic solutions of the Korteweg-de Vries equation and a related many-body problem. Comm. Pure Appl. Math., 30:95-148, 1977.

[4] P.J. Angulo, J.L. Bona, and M. Scialom. Stability of cnoidal waves. Adv. Differential Equations, 11:1321-1374, 2006.

[5] V. I. Arnold. Mathematical methods of classical mechanics. Springer-Verlag, New York, NY, 1997.

[6] V. I. Arnold. On an a priori estimate in the theory of hydrodynamical stability. Am. Math. Soc. Tranl., 79, 267-269, 1969.

[7] E. D. Belokolos, A. I. Bobenko, V. Z. Enol'skii, A. R. Its, and V. B. Matveev. Algebro-geometric approach to nonlinear integrable problems. Springer-Verlag, Berlin, 1994. 
[8] T. B. Benjamin. The stability of solitary waves. Proc. Roy. Soc. London Ser. A, 328:153-183, 1972.

[9] O. I. Bogojavlenskiu and S. P. Novikov. The connection between the Hamiltonian formalisms of stationary and nonstationary problems. Funct. Anal. Appl., 10:8-11, 1976.

[10] H. Bohr. Almost Periodic Functions. Chelsea Publishing Company, New York, N.Y., 1947.

[11] J. Bona. On the stability theory of solitary waves. Proc. Roy. Soc. London Ser. A, 344:363-374, 1975.

[12] N. Bottman and B. Deconinck. KdV cnoidal waves are spectrally stable. DCDS-A, 25:11631180, 2009.

[13] J.C. Bronski and M.A. Johnson. The modulational instability for a generalized KdV equation. To appear in the Archive for Rational Mechanics and Analysis, 2009.

[14] J.C. Bronski, M.A. Johnson, and T. Kapitula. An index theorem for the stability of periodic traveling waves of KdV type. Submitted for Publication, 2009.

[15] P. F. Byrd and M. D. Friedman. Handbook of elliptic integrals for engineers and scientists. Springer-Verlag, New York, NY, 1971.

[16] B. Deconinck and T. Kapitula. On the orbital (in)stability of spatially periodic stationary solutions of generalized Korteweg-de Vries equations. Submitted for publication, 2009.

[17] B. Deconinck, F. Kiyak, J. D. Carter, and J. N. Kutz. Spectruw: a laboratory for the numerical exploration of spectra of linear operators. Mathematics and Computers in Simulation, 74:370$379,2007$.

[18] B. Deconinck and J. N. Kutz. Computing spectra of linear operators using Hill's method. Journal of Computational Physics, 219:296-321,2006.

[19] B. Deconinck and H. Segur. Pole dynamics for elliptic solutions of the Korteweg-de Vries equation. Math. Phys. Anal. Geom., 3:49-74, 2000.

[20] L. A. Dickey. Soliton equations and Hamiltonian systems. World Scientific Publishing Co. Inc., River Edge, NJ, second edition, 2003.

[21] B. A. Dubrovin. Theta functions and nonlinear equations. Russian Math. Surveys, 36:11-80, 1981.

[22] B. A. Dubrovin and S. P. Novikov. Periodic and conditionally periodic analogs of the manysoliton solutions of the Korteweg-de Vries equation. Soviet Phys. JETP, 40:1058, 1974.

[23] V. Z. Enol'skii. On solutions in elliptic functions of integrable nonlinear equations associated with two-zone Lamé potentials. Soviet Math. Dokl., 30:394-397, 1984.

[24] H. M. Farkas and I. Kra. Riemann surfaces. Springer-Verlag, New York, NY, 1992.

[25] C. S. Gardner. The Korteweg-de Vries equation and generalizations. IV. The Korteweg-de Vries equation as a Hamiltonian system. J. Mathematical Phys., 12:1548-1551, 1971. 
[26] M. Grillakis, J. Shatah, and W. Strauss. Stability theory of solitary waves in the presence of symmetry. I. J. Funct. Anal., 74:160-197, 1987.

[27] M. Grillakis, J. Shatah, and W. Strauss. Stability theory of solitary waves in the presence of symmetry. II. J. Funct. Anal., 94:308-348, 1990.

[28] M. Haragus and T. Kapitula. On the spectra of periodic waves for infinite-dimensional Hamiltonian systems. Physica D, 237 (2008) 2649-2671.

[29] D. B. Henry, J. F. Perez, and W. F. Wreszinski. Stability theory for solitary-wave solutions of scalar field equations. Comm. Math. Phys., 85:351-361, 1982.

[30] D. D. Holm, J. E. Marsden, T. Ratiu, and A. Weinstein. Nonlinear stability of fluids and plasma equilibria. Physics Reports, 123:1-116, 1985.

[31] E. L. Ince. Further investigations into the periodic Lamé functions. Proc. Roy. Soc. Edinburgh, 60:83-99, 1940.

[32] E. L. Ince. Ordinary Differential Equations. Dover Publications, New York, 1944.

[33] E. Infeld and G. Rowlands. Nonlinear waves, solitons and chaos. Cambridge University Press, Cambridge, second edition, 2000.

[34] A. R. Its and V. B. Matveev. Schrödinger operators with the finite-band spectrum and the $N$-soliton solutions of the Korteweg-de Vries equation. Theoret. and Math. Phys., 23:343-355, 1976.

[35] M.A. Johnson. Nonlinear stability of periodic traveling wave solutions of the generalized Korteweg-de Vries equation. SIAM Journal on Mathematical Analysis, 41:1921-1947, 2009.

[36] T. Kapitula. On the stability of $N$-solitons in integrable systems. Nonlinearity, 20:879-907, 2007.

[37] T. Kapitula, P. G. Kevrekidis, and B. Sandstede. Counting eigenvalues via the Krein signature in infinite-dimensional Hamiltonian systems. Phys. D, 195:263-282, 2004.

[38] D.J. Korteweq and G. de Vries. On the change of form of long waves advancing in a rectangular channel, and on a new type of long stationary waves. Philosophical Magazine, 39:422-443, 1895.

[39] I. M. Krichever. Methods of algebraic geometry in the theory of nonlinear equations. Russian Math. Surveys, 32:185-213, 1977.

[40] M. D. Kruskal, R. M. Miura, C. S. Gardner, and N. J. Zabusky. Korteweg-de Vries equation and generalizations. V. Uniqueness and nonexistence of polynomial conservation laws. $J$. Mathematical Phys., 11:952-960, 1970.

[41] D. F. Lawden. Elliptic functions and applications. Springer-Verlag, New York, NY, 1989.

[42] P. D. Lax. Periodic solutions of the KdV equation. Comm. Pure Appl. Math., 28:141-188, 1975 . 
[43] J. H. Maddocks and R. L. Sachs. On the stability of KdV multi-solitons. Comm. Pure Appl. Math., 46:867-901, 1993.

[44] W. Magnus and S. Winkler. Hill's equation. Dover Publications Inc., New York, NY, 1979.

[45] H. P. McKean. Stability for the Korteweg-deVries equation. Comm. Pure and Appl. Math., 30:347-353, 1977.

[46] H. P. McKean and E. Trubowitz. Hill's operator and hyperelliptic function theory in the presence of infinitely many branch points. Comm. Pure Appl. Math., 29:143-226, 1976.

[47] S. Novikov, S. V. Manakov, L. P. Pitaevskiı, and V. E. Zakharov. Theory of solitons. New York, NY, 1984.

[48] S. P. Novikov. A periodic problem for the Korteweg-de Vries equation. I. Funct. Anal. Appl., 8:236-246, 1974.

[49] P. J. Olver. Applications of Lie groups to differential equations. Springer-Verlag, New York, 1993.

[50] M. Sato and Y. Sato. Soliton equations as dynamical systems on infinite-dimensional Grassmann manifold. In Nonlinear partial differential equations in applied science (Tokyo, 1982), pages 259-271. North-Holland, Amsterdam, 1983.

[51] G. Springer. Introduction to Riemann surfaces. Addison-Wesley Publishing Company, Inc., Reading, Mass., 1957.

[52] M. I. Weinstein. Lyapunov stability of ground states of nonlinear dispersive evolution equations. Comm. Pure Appl. Math., 39:51-67, 1986.

[53] V.E. Zakharov and L.D. Faddeev. Korteweg-de Vries equation, a completely integrable Hamiltonian system. Funct. Anal. Appl., 5:280-287, 1971. 